\title{
Ecología trófica del barbo (Barbus sclateri) en cuatro embalses del Sur de España
}

\author{
L. Encina, V. Castaño, B. García \& M. Gil \\ Depto Biología Vegetal y Ecología. Fac. Biología. Univ. Sevilla. Apdo. 1095. 41080 Sevilla, España.
}

\section{RESUMEN}

Los objetivos principales del presente estudio fueron conocer las estrategias tróficas del barbo de Esclater (Barbus sclateri, Günther, 1868), en cuatro embalses de abastecimiento de agua potable, localizados en el Río Rivera de Huelva, en el sur de la Península Ibérica: Aracena, Zufre, La Minilla y El Gergal. El estudio se llevó a cabo durante el invierno, coincidiendo con el periodo de mezcla de la masa de agua en los cuatro embalses. La dieta de los ejemplares procedentes de Aracena, Zufre y Minilla resultó ser muy similar, quedando la de El Gergal separada en función de los análisis efectuados. Los elementos que determinan esta separación en las dietas son la presencialausencia de componentes animales (zooplancton o macroinvertebrados) y restos vegetales, y la incidencia del detrito. Pese a estas diferencias en la composición de la dieta, la estrategia trófica desarrollada por la especie es la misma en los cuatro embalses, caracterizada por el consumo de una gran variedad de elementos (omnivoría, eurifagia), la mayoría de ellos sin dominancia clara sobre los otros (generalismo), y una amplitud del nicho trófico caracterizado por una alta componente intrafenotípica.

Palabras claves: Alimentación, barbo, embalses.

\begin{abstract}
The main objectives of the present study were to examine the feeding habits of Esclater's barbel (Barbus sclateri, Günther, 1868), in four reservoirs for drinking water supply, located along the course of the river Rivera in Huelva (South of the Iberian Peninsula). The reservoirs are called Aracena, Zufre, The Minilla and The Gergal. The study was carried out in winter, when all four reservoirs were well-mixed. The diet of barbel in Aracena, Zufre and Minilla reservoirs proved to be very similar, as opposed to barbel in El Gergal. This difference in diets across reservoirs was apparently determined by the presence/absence of animal components (i.e. zooplankton or macroinvertebrates), plant remains, and detritus. In spite of these differences in diet composition, the feeding strategy developed by barbel was similar in the four reservoirs. A great variety of items (omnivory, eurifagy) were ingested by barbel in the four reservoirs without any clear preference for any (i.e. generalism). The trophic niche of barbel has a high intraphenotypic component.
\end{abstract}

Key-words: Barbel, feeding, reservoirs.

\section{INTRODUCCIÓN}

Las estrategias tróficas desarrolladas por las especies han despertado un creciente interés en los últimos años a partir del desarrollo de la teoría del optimal foraging (Schoener, 1971; Kamil et al., 1987). Por otra parte, determinar los consumos alimentícios de las especies es de especial interés en las implicaciones top-down vs bottom-up dentro del ecosistema, y pueden ser especialmente útiles en modelos de gestión de los mismos (Allen \& Wootton, 1982; Stewart et al., 1983). En el marco de éstos surge el presente trabajo, cuyos objetivos principales fueron conocer las estrategias tróficas, y estimar las tasas de consumo alimentício del barbo de 


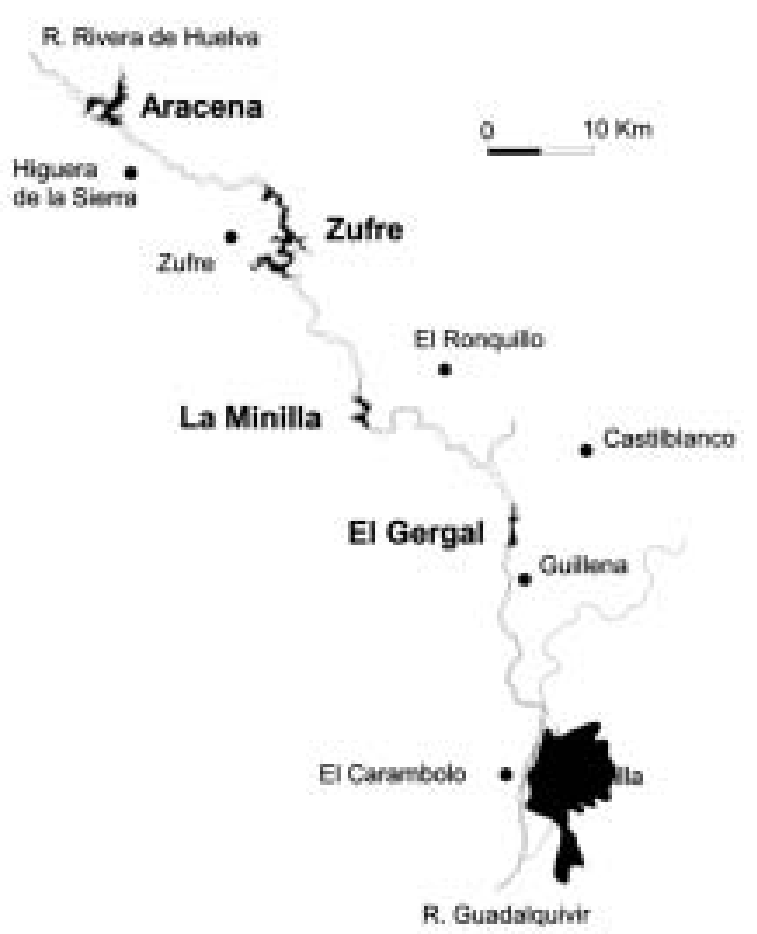

Figura 1. Área de estudio. Study area

Esclater (Barbus sclateri, Günther 1868), en cuatro embalses de abastecimiento de agua potable, localizados en el Río Rivera de Huelva, en el sur de la Península Ibérica.

El barbo de Esclater, y en general todas las especies de barbo, ha sido usualmente descrito desde el punto de vista trófico como generalista, con una dieta amplia y oportunista (Granado-Lorencio, 1983; Encina \& Granado Lorencio, 1988; Encina, 1991; Magalhaes, 1992, 1993). Esta descripción ha sido basada siempre sobre estudios de la población, nunca sobre estrategias alimentícias individuales de cada miembro de la misma. Es evidente que una población con un nicho trófico estrecho, necesariamente está compuesta por individuos que a su vez poseen nichos tróficos igualmente estrechos y especializados. Sin embargo, una población que presente un amplio nicho trófico puede estar formada por individuos tanto con un amplio nicho trófico, como por nichos tróficos individualmente estrechos y especializados,

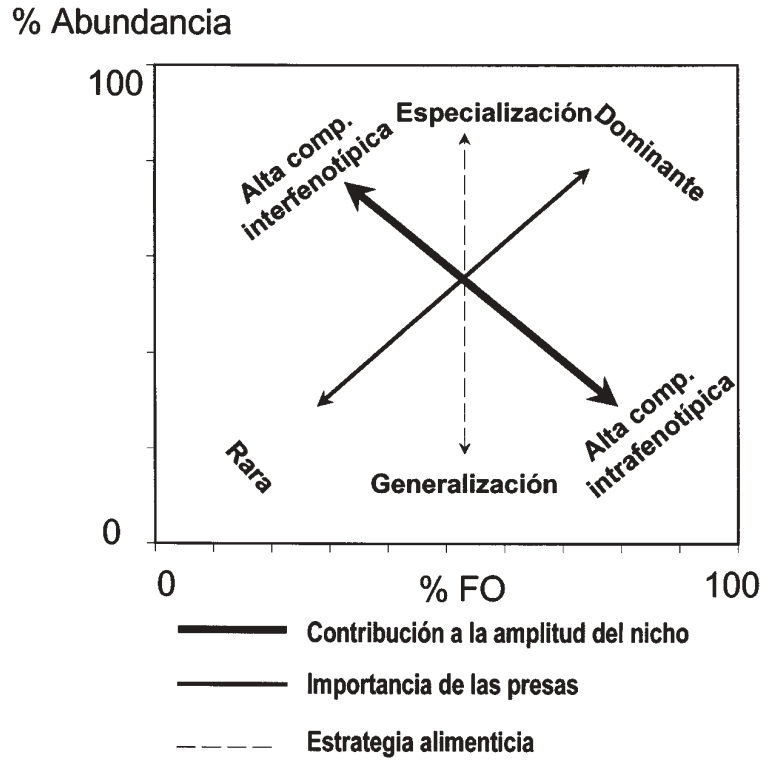

Figura 2. Evaluación gráfica de la estrategia alimenticia de Amundsen et al. (1996). Graphic evaluation of feeding strategy (Amundsen et al., 1996).

o una combinación de ambos casos (Amundsen, 1995). Esta diferencia puede resultar relevante en la explotación del ecosistema y en las relaciones bióticas del mismo.

\section{MATERIAL Y MÉTODOS}

Los cuatro embalses estudiados son: Aracena $\left(128 \mathrm{hm}^{3}\right)$, Zufre $\left(168 \mathrm{hm}^{3}\right)$, La Minilla $\left(58 \mathrm{hm}^{3}\right)$ y El Gergal $\left(35 \mathrm{hm}^{3}\right)$, ubicados en cadena en el Río Rivera de Huelva (Fig. 1). Dichos embalses presentan una eutrofia moderada, y un ciclo de estratificación termal entre los meses de Mayo a Octubre, con termoclina estable entre los 6-8 m de profundidad. El grado de mineralización es bajo, como corresponde a la naturaleza silicea de la cuenca. Entre las especies que componen la asociación íctica de estos embalses encontramos únicamente dos especies endémicas, Barbus sclateri y Chondrostoma polylepis, siendo las restantes especies introducidas (Cyprinus carpio, 
Micropterus salmoides, Lepomis gibbosus y Gambusia holbrooki).

El estudio se llevó a cabo durante el invierno, coincidiendo con el periodo de mezcla de la masa de agua en los cuatro embalses. Los peces fueron capturados empleando artes de atrapamiento (trasmallos y redes holandesas). Todos los ejemplares seleccionados estuvieron comprendidos en un estrecho rango de tallas $(325-351 \mathrm{~mm})$ y peso (350-425 g), a fin de evitar influencias de tipo ontogénico en los resultados. El total de individuos capturados fue de 62 ejemplares. En cada individuo se midió la longitud total y estándar, se pesó (peso fresco y peso seco), y se diseccionó para extraer la totalidad del tubo digestivo (al no existir un estómago diferenciado y un ciclo alimenticio largo y continuo, con una digestión muy lenta; Encina \& Granado-Lorencio, 1991, 1994). Los contenidos fueron removidos y congelados para su análisis. El periodo de manipulación previo a la congelación de las muestras no excedió en ningún caso las 4 horas. La determinación de la dieta se realizó mediante análisis cualitativo y cuantitativo de los contenidos gastrointestinales. Para el análisis cuantitativo se empleó el método volumétrico de Larimore, recopilado en Amezaga-Herrán (1988). Se emplearon en el análisis las medidas de frecuencia de ocurrencia $\left(\mathrm{F}_{\mathrm{i}}\right)$ y frecuencia de abundancia $\left(\mathrm{A}_{\mathrm{i}}\right)$ de cada una de las presas encontradas (Hyslop, 1980). Se determinó también la frecuencia de abundancia específica $\left(\mathrm{P}_{\mathrm{i}}\right)$ (Amundsen et al., 1996). En términos matemáticos, estas expresiones quedan descritas por las ecuaciones:

$$
\begin{aligned}
F_{i} & =N_{i} / N \\
A_{i} & =\left(\Sigma S_{i} / \Sigma S t\right) \times 100 \\
P_{i} & =\left(\Sigma S_{i} / \Sigma S t_{i}\right) \times 100
\end{aligned}
$$

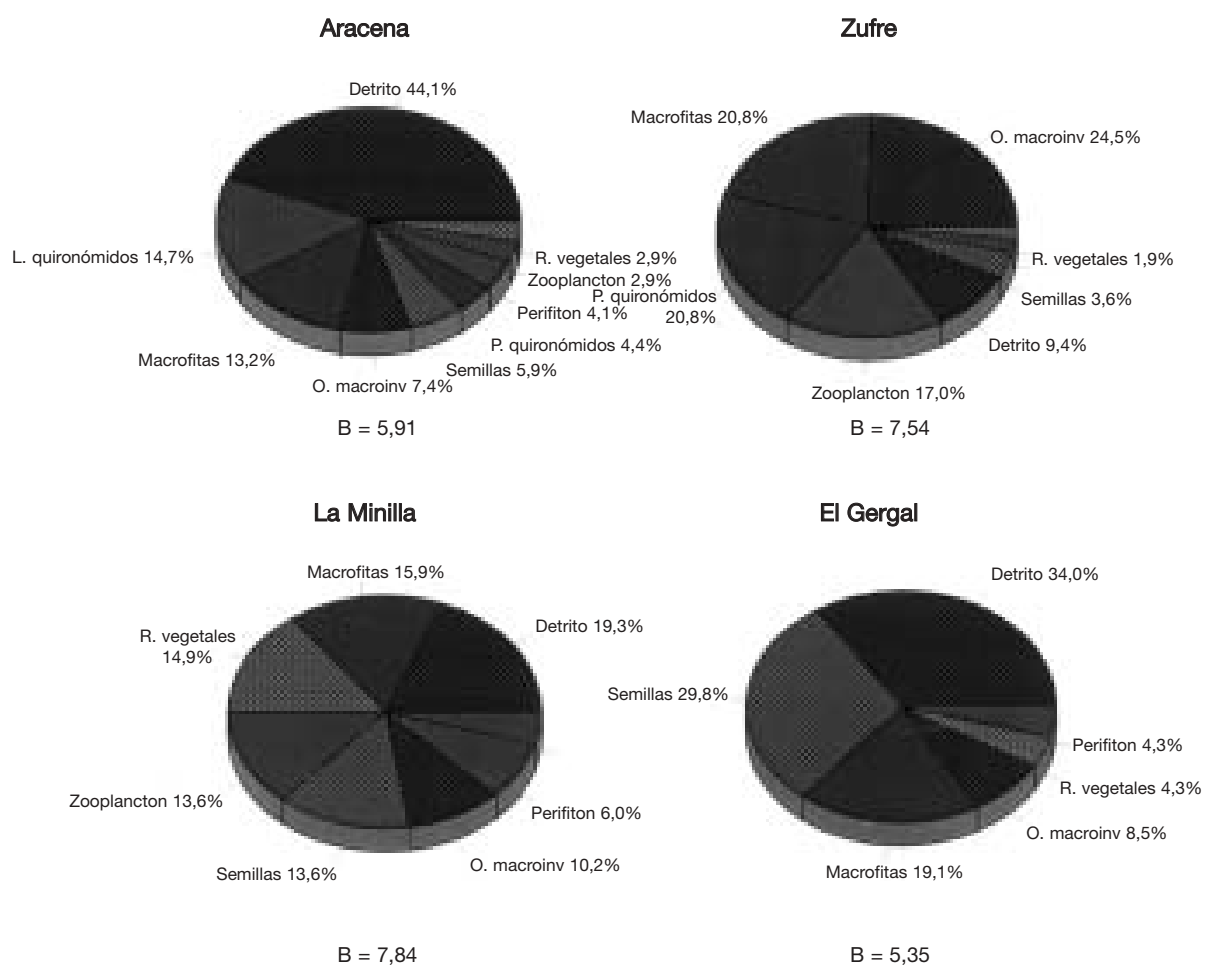

Figura 3. Composición de la dieta (en \% de abundancia) encontrada en cada uno de los embalses, y amplitud del nicho trófico (índice B de Levins). (R: restos; P: pupas; L: larvas; O: otros). Diet composition (in \% abundance) of barbel found in four reservoirs, and width of the trophic niche (i.e. index B of Levins). (R: remains; P: pupae; L: larvae; O: others). 
donde $N_{i}$ es el número de ejemplares con presa $i$ en el contenido gastrointestinal, $N$ el número total de individuos, $S_{i}$ la abundancia de la presa $i, S t$ la abundancia de los contenidos gastrointestinales totales, y $S t_{i}$ la abundancia total de los contenidos gastrointestinales con presa $i$.

Se analizaron tanto la composición cualitativa y cuantitativa de la dieta, como el contenido energético de la misma, y las variaciones de éstos en el ciclo anual, y entre los distintos embalses. Para la caracterización de las dietas se empleó el índice de importancia en la alimentación de Windell (1971),

$$
I I A=\sqrt{ }\left(F_{i} \times A_{i}\right)
$$

La estrategia alimentícia se estudió a nivel tanto poblacional como individual, usando un nuevo método gráfico de evaluación (Fig. 2) (Amundsen et al., 1996). El análisis gráfico consiste en representar, para cada componente de la dieta, su frecuencia de ocurrencia frente a su abundancia específica. La importancia de cada presa, la estrategia alimenticia y la contribución fenotípica a la amplitud del nicho trófico puede ser interpretada a lo largo de las diagonales generadas en el gráfico.

Como medida de la amplitud del nicho trófico se empleó el índice estandarizado de Levins (Krebs, 1998):

$$
\mathrm{B}=1 / \Sigma \mathrm{p}_{\mathrm{i}}^{2}, \mathrm{i}=1 \ldots \mathrm{n}
$$

donde $p_{i}$ es la fracción de la presa $i$ en la dieta $\mathrm{y}$ $n$ el número total de elementos de la dieta.

Para estimar las tasas de consumo alimenticio se empleó el modelo bioenergético propuesto por Penczak (1990, 1995). Estos se expresaron como Ración Diaria (RD), es decir, los gramos de alimento consumidos expresados como porcentaje del peso corporal del individuo. Para determinar el equivalente calórico de la dieta en cada embalse se utilizó un calorímetro Parr modelo 1341. Para la calibración del calorímetro se emplearon pastillas de ácido benzoico con un calor de combustión de $6318 \mathrm{cal} / \mathrm{g}$, correspondiendo el equivalente energético del calorímetro a un valor de $2519.5 \mathrm{cal} /{ }^{\circ} \mathrm{C}$.

Para el tratamiento estadístico de los resultados obtenidos se emplearon, en el caso de los componentes de la dieta, los test de KruskalWallis y U de Mann Whitney; y en el caso del contenido energético y de las estimas de consumo alimenticio, se utilizó el análisis de la varianza (ANOVA), previa verificación de las condiciones de normalidad y homocedasticidad de las mues-

Tabla 1. Resultados del análisis de Kruskal-Wallis sobre la incidencia de los distintos tipos de presa en la dieta para los cuatro embalses. (L: larvas; P: pupas; O: otros; R: restos). Results of the KruskalWallis analysis carried out to test differences in the food item presence in the diet of barbel from four South Spain reservoirs (Aracena, Zufre, La Minilla and El Gergal). (L: larvae; P: pupae; $O$ : others; $R$ : remains).

\begin{tabular}{lrrc}
\hline & df & $\boldsymbol{\chi}^{\mathbf{2}}$ & $\mathbf{p}$ \\
\hline Zooplancton & 3 & 14,00 & 0,0029 \\
L.quironómidos & 3 & 8,24 & 0,0412 \\
P.quironómidos & 3 & 8,55 & 0,0359 \\
O.macro & 3 & 6,14 & 0,1048 \\
invertebrados & & & \\
Perifiton & 3 & 3,29 & 0,3488 \\
Semillas & 3 & 10,80 & 0,0128 \\
R.vegetales & 3 & 17,62 & 0,0005 \\
Macrófitas & 3 & 0,21 & 0,9747 \\
Detrito & 3 & 17,32 & 0,0006 \\
\hline
\end{tabular}

Tabla 2. Resultados del análisis U de Mann-Whitney para los tipos de presa significativamente distintos $(\mathrm{p}<0,01)$ en la dieta para los cuatro embalses estudiados. En negrilla se indican las diferencias significativas para $\mathrm{p}<0,01$. Results of the Mann-Whitney $U$ test analysis carried out for food items present at statistically different proportions $(p<0.01)$ in the diet of barbel from the four reservoirs. Bold characters indicate significant differences at $p<0.01$.

\begin{tabular}{lccc}
\hline Zooplancton & $\begin{array}{c}\text { Aracena } \\
(\mathbf{N}=\mathbf{1 7})\end{array}$ & Zufre & Minilla \\
\hline Zufre $(\mathrm{N}=15)$ & $\mathbf{2 6 . 0}$ & & \\
Minilla $(\mathrm{N}=16)$ & $\mathbf{1 2 . 5}$ & 35.5 & \\
Gergal $(\mathrm{N}=14)$ & $\mathbf{2 8 . 0}$ & $\mathbf{1 7 . 5}$ & $\mathbf{3 . 5}$ \\
\hline R.vegetales & Aracena & Zufre & Minilla \\
Zufre & 36.0 & & \\
Minilla & $\mathbf{9 . 5}$ & $\mathbf{7 . 0}$ & \\
Gergal & 30.0 & $\mathbf{3 0 . 0}$ & 10.0 \\
\hline Detrito & Aracena & Zufre & Minilla \\
Zufre & $\mathbf{1 . 0}$ & & \\
Minilla & $\mathbf{1 1 . 0}$ & 22.0 & \\
Gergal & $\mathbf{1 1 . 0}$ & 14.5 & 30.0 \\
\hline
\end{tabular}




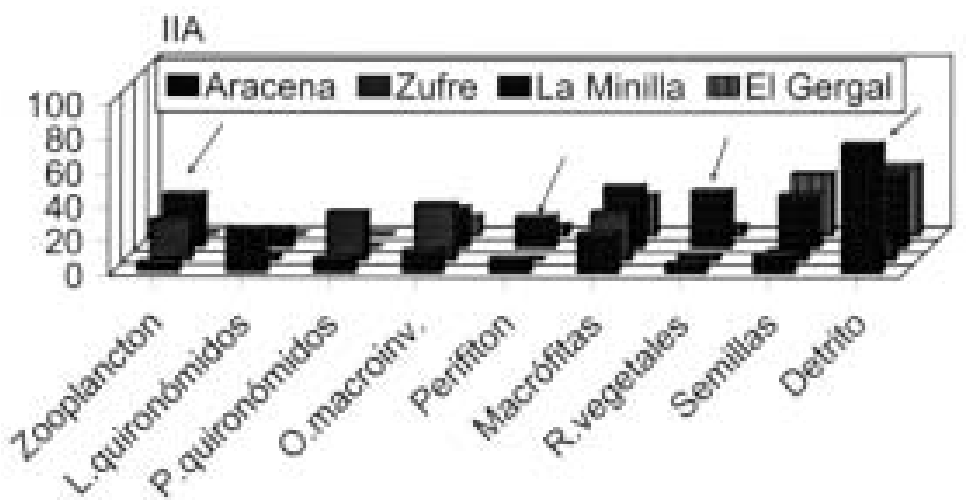

Figura 4. Valores para el índice de importancia alimenticia (IIA) de los distintos elementos de la dieta encontrados en el barbo para cada uno de los embalses. Las flechas indican diferencias significativas a P < 0,01. (R: restos; P: pupas; L: larvas; O: otros). Value for the index of feeding importance (IIA) of the different elements of the diet of barbel in the four reservoirs. Arrows indicate significant differences at $p<0.01$. (R: remains; $P$ : pupae; L: larvae; O: other).

tras (test de Kolmogorov-Smirnov, test de Cochrans y test de Bartlett-Box). En todas las comparaciones las muestras mostraron normalidad y homocedasticidad. Las diferencias entre cada par de embalse se contrastaron mediante el test de Tukey. En todos los casos una probabilidad del 0.01 fue utilizada para contrastar la significación de la hipótesis nula.

El análisis de cluster categóricos y el análisis de función discriminante categórico (procedimiento CATMOD) fueron empleados para analizar la similaridad de la composición cualitativa y cuantitativa de la dieta en los diferentes embalses. Los análisis se llevaron a cabo empleando el programa SAS. Las categorías asignadas para los análisis fueron: ausente = categoría $0 ;<25 \%$ de abundancia $=$ categoría 1 ; entre el 25 y el $50 \%$ de abundancia $=$ categoría 2 ; entre el 50 y el $75 \%=$ categoría 3; mas del 75\% = categoría 4 .

\section{RESULTADOS}

Si bien los elementos integrantes en la dieta para los cuatro embalses son prácticamente los mismos (Fig. 3), se registran diferencias significativas en la composición cualitativa y cuantitativa de los mismos (Tablas 1 y 2). Estas diferencias registradas entre los distintos embalses se refieren principalmente a la incidencia en la dieta de detrito, en contraposición con la presencia de restos vegetales y crustaceos zooplanctónicos $(\mathrm{p}<$ 0.01 ); y secundariamente a la importancia en la misma de las larvas y pupas de quironómidos, y las semillas $(p<0.05)$. La dieta del barbo en Aracena se caracteriza por un mayor consumo de detrito que en los restantes embalses (Fig. 4); en La Minilla, es el consumo significativamente mayor de restos vegetales el que caracteriza la dieta, junto con una mayor incidencia (salvo respecto a Zufre) de crustaceos zooplanctónicos. Éstos últimos, junto con la mayor presencia de pupas de quironómido, son las diferencias destacables de la dieta en el caso de Zufre. Por último, no existe ningún componente tipificador para la

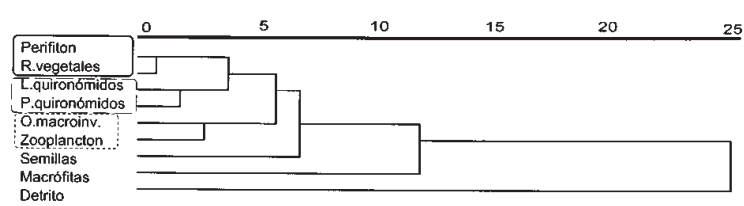

Figura 5. Resultados del análisis de agrupamiento efectuado sobre los distintos componentes de la dieta para el conjunto de todos los individuos, independientemente del embalse de procedencia. (R: restos; P: pupas; L: larvas; O: otros). Results of the cluster analysis on the different components of the diet of barbel (all data). (R: remains; P: pupae; L: larvae; $O$ : others). 
Tabla 3. Resultados del análisis categórico (CATMOD) de los componentes de la dieta para los cuatro embalses. Results of the categorical (CATMOD) analysis carried out for food items in the barbel diet from the four reservoirs.

\begin{tabular}{ccccccc}
\hline Función canónica & Eigenvalue & \% varianza & I Wilks & $\boldsymbol{\chi}^{2}$ & df \\
\hline 1 & 5.71 & 66.85 & 0.0259 & 98.61 & 30 \\
2 & 1.73 & 20.29 & 0.1741 & 47.19 & 18 \\
3 & 1.10 & 12.86 & 0.4762 & 20.03 & 80.001 & 8
\end{tabular}

Coeficientes estandarizados para las funciones discriminantes canónicas.

\begin{tabular}{ccccc}
\hline & Función 1 & Función 2 & Función 3 \\
\hline Detrito & 0.0979 & 0.9954 & 0.0765 & \\
Semillas & 0.2567 & -0.3856 & 0.9314 & \\
R.vegetales & 0.9649 & 0.1077 & -0.3321 & \\
& \multicolumn{5}{c}{} \\
Porcentaje (\%) evaluado correctamente en el grupo clasificado. \\
\multicolumn{5}{c}{ Grupo predicho } \\
Grupo actual & Aracena & Zufre & La Minilla & El Gergal \\
Aracena & 88.9 & 0 & 0 & 11.1 \\
Zufre & 10.0 & 0 & 90 & 0 \\
La Minilla & 0 & 100 & 0 & 0 \\
El Gergal & 0 & 0 & 14.3 & 85.7 \\
\hline
\end{tabular}

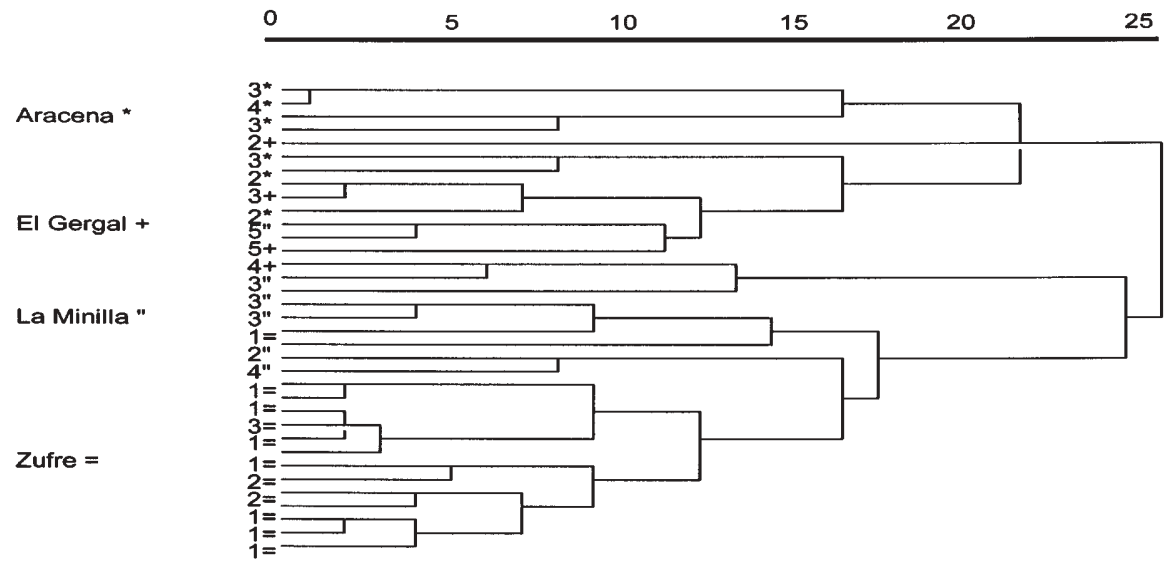

Figura 6. Resultados del análisis de agrupamiento efectuado sobre los ejemplares capturados en cada uno de los embalses. Results of the cluster analysis on individuals captured in the four reservoirs.

dieta en El Gergal, pero destaca por ser la de menor amplitud del nicho trófico (B en la Fig. 3).

Si efectuamos una clasificación por técnicas de agrupamiento de los distintos elementos encontrados en la dieta (usando como entrada la matriz de contenidos estomacales individuales y tomando como variables los elementos de la dieta), independientemente del embalse de procedencia de la muestra, encontramos que existen cuatro elementos muy relacionados, con una 
clara componente indicativa de hábitos bentófagos: las larvas y pupas de quironómidos, y el binomio perifiton-restos vegetales (Fig. 5). Otro grupo que guarda cierta relación o cercania es el par zooplancton - otros macroinvertebrados, el cual incluye mayoritariamente invertebrados de origen terrestre que suelen caer a la superficie del agua donde son consumidos. Este segundo grupo representaría una componente trófica en relación al hábitat trófico opuesto al primero, es decir, una alimentación planctófaga o limnética. Por último, y muy distanciado de los restantes elementos, aparece el detrito. Este "aislamiento" de los restantes componentes indica que este elemento de por sí se asocia a un tipo de régimen trófico característico, el detritívoro, en el que este elemento es dominante o principal, y el resto de elementos que en un momento dado pueden ser consumidos, lo hacen en forma adicional o esporádica.

Cuando el análisis lo realizamos sobre los casos, es decir, clasificando o agrupando los individuos (igualmente los contenidos estomacales se analizan individualmente) en relación al embalse de procedencia, encontramos que, con las dos técnicas empleadas, los ejemplares procedentes de Aracena, Zufre y Minilla quedan perfectamente separados en función de su dieta (Figs. 6 y 7),

Tabla 4. Resultados del análisis de la varianza (One-Way ANOVA) y del test de Tukey para el contenido energético de la dieta en los cuatro embalses. (X: contenido energético medio, en cal; Los asteriscos indican diferencias significativas entre pares cuando están en posiciones diferentes). One-Way ANOVA results and Tukey tests carried out for the caloric content of the diet in the four reservoirs. (X: mean caloric content, cal; asterisk designates statistical differences between pairs).

\begin{tabular}{llcc}
\hline & df & F & p \\
\hline Factor Embalse & 3.31 & 10.54 & $<0.001$ \\
& X (cal) & Test de & Tukey \\
\hline $\begin{array}{l}\text { Aracena } \\
\text { Lafre } \\
\text { El Gergal }\end{array}$ & 1167.44 & $\#$ & \\
& 3233.78 & & $\#$ \\
\hline
\end{tabular}

no así los de El Gergal. Los elementos que determinan esta separación son nuevamente, la presencia de restos vegetales, que aporta el mayor coeficiente al eje canónico I (Tabla 3), y la incidencia del detrito, que determina el eje canónico II, Entre ambos ejes se absorbe un $87.14 \%$ de la varianza, y se clasifican correctamente dentro de cada grupo más del $85 \%$ de los casos.

Pese a estas diferencias en la composición de la dieta, la estrategia trófica desarrollada por la especie es la misma en los cuatro embalses, caracterizada por el consumo de una gran variedad de elementos (omnivoría, eurifagia), la mayoría de ellos sin dominancia clara sobre los otros (generalismo), y una amplitud del nicho trófico caracterizado por una alta componente intrafenotípica (Fig. 8). Esto significa que todos los individuos de la población participan o contribuyen cuantitativa y cualitativamente de forma similar al nicho trófico, no registrándose especializaciones de tipo individualistas, es decir, todos comen un poco de todo (de todos los elementos que componen el nicho trófico del conjunto de la población).

En cuanto al contenido energético, se han registrado diferencias significativas en el equivalente calórico de las dietas entre los embalses de Aracena-Minilla y Zufre-Gergal (Tabla 4), siendo significativamente mayor en los dos últi-

Tabla 5. Resultados del análisis de la varianza (One-Way ANOVA) y del test de Tukey para la tasa de consumo alimenticio (RD) en los cuatro embalses. (X: Ración diaria media, en \% del peso corporal; Los asteriscos indican diferencias significativas entre pares cuando están en posiciones diferentes). One-Way ANOVA results and Tukey tests carried out for the food consumption rate (RD) estimated in the four reservoirs. ( $X$ : mean daily ration, expressed as \% of body weight; asterisk designates statistical differences between pairs).

\begin{tabular}{llcc}
\hline & df & F & p \\
\hline Factor Embalse & 3.31 & 232.44 & $<0.001$ \\
& X (cal) & Test de & Tukey \\
\hline Aracena & 2.5 & $\#$ & \\
Zufre & 0.7 & & $\#$ \\
La Minilla & 1.3 & $\#$ & \\
El Gergal & 0.8 & & $\#$ \\
\hline
\end{tabular}




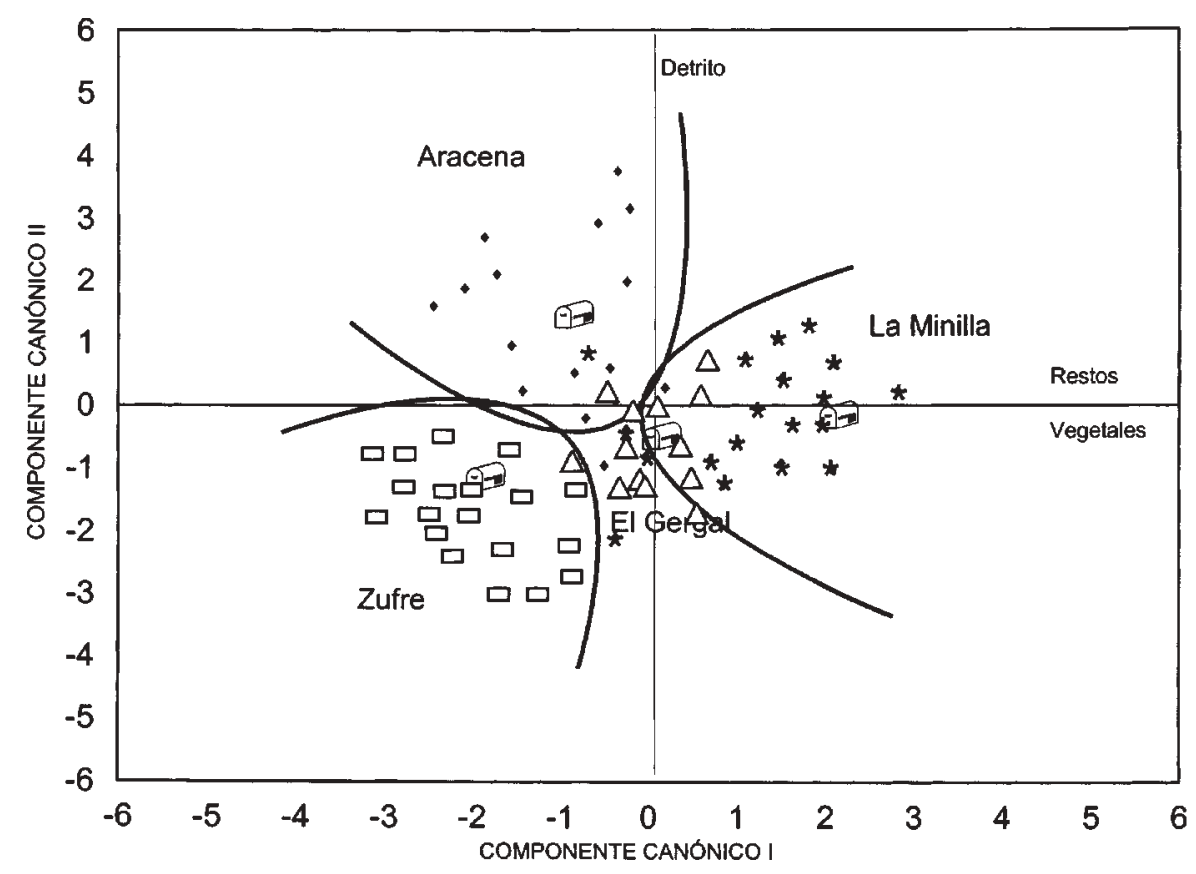

Figura 7. Distribución de los individuos pertenecientes a los distintos embalses en el espacio definido por los componentes canónicos I y II del análisis discriminante. Distribution of individual barbe of the four reservoirs in the space defined by canonical components I and II of the discriminant analysis

mos. Las diferencias registradas en la composición cualitativa y cuantivativa de la dieta entre los embalses serían la causa de las diferencias en el contenido energético de las mismas, que a su vez determinarían las diferencias significativas en las tasas de consumo alimenticio (expresada en forma de ración diaria, RD) estimada para cada embalse (Tabla 5).

\section{DISCUSIÓN}

La composición de la dieta del barbo de Esclater en estos cuatro embalses confirma la estrategia generalista desarrollada por esta especie. Las presas ingeridas pertenecen tanto a zooplancton, como zoobentos, insectos terrestres, plantas, detrito, etc. La amplitud del nicho trófico y los componentes del mismo son prácticamente iguales en los cuatro embalses estudiados, únicamente varían los componentes en relación a su proporciones dentro de la dieta. Esto significa que esencialmente se explotan los mismos recursos, variando la proporción de los mismos en función probablemente de su abundancia en cada uno de estos embalses mas, que reflejar pautas o hábitos alimenticios distintos en cada uno de ellos. En todos los embalses el nicho trófico es amplio $(\mathrm{B}>5)$, tanto a nivel poblacional como individual, como demuestra la alta componente intrafenotípica del mismo.

En todos los embalses la dieta presentó una componente animal estrecha, principalmente constituida por larvas y pupas de quironómidos, las presas más abundantes en el bentos de los embalses. Estos resultados corroboran los encontrados por Lobón-Cerviá \& Diego (1988), Encina (1986, 1991), o Encina \& Granado-Lorencio $(1994,1997)$ en el sentido de que el barbo tiende a alimentarse de aquellas presas animales disponibles en el medio más dominantes y de mas facil 

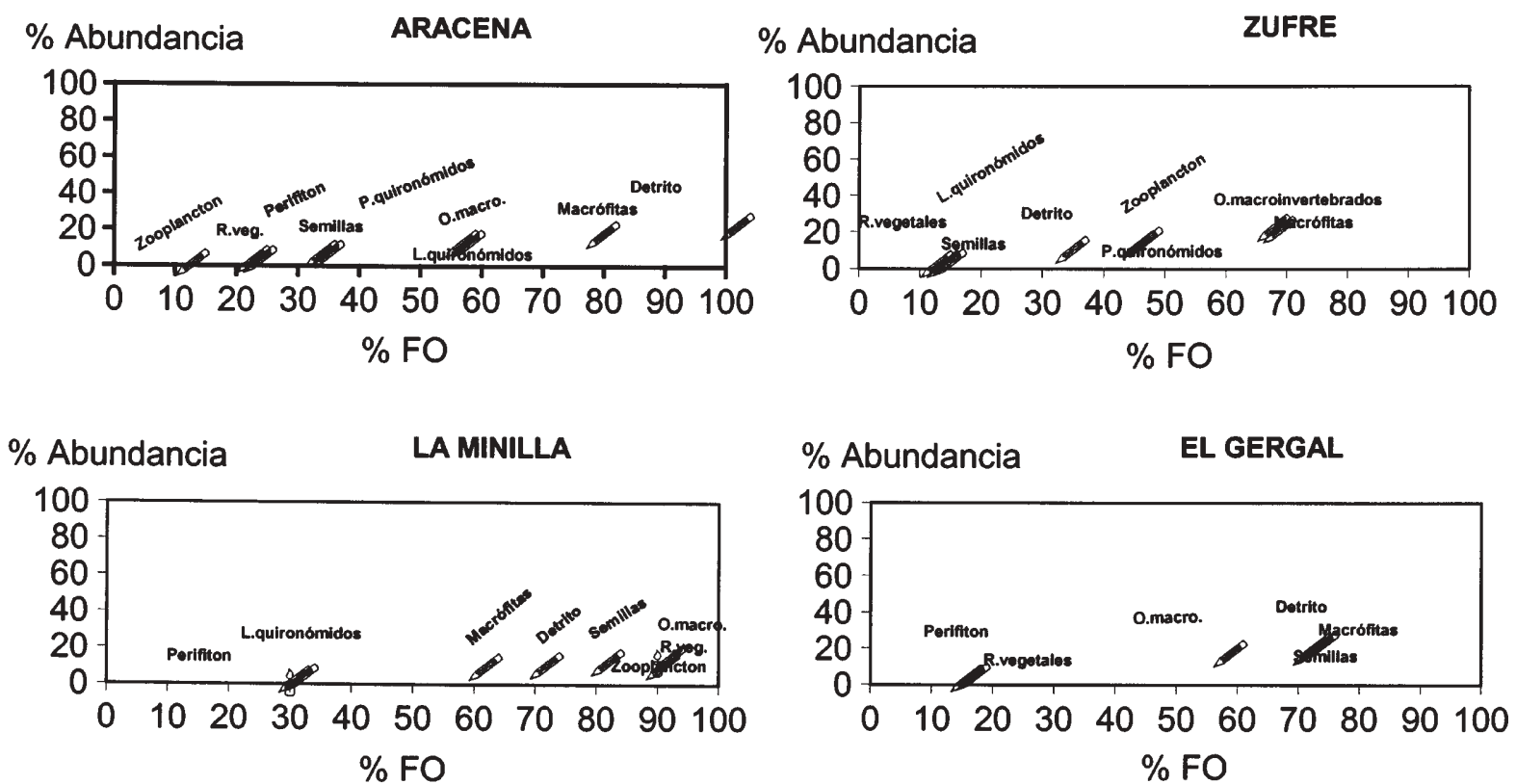

Figura 8. Representación de las estrategias alimenticias según el método gráfico de Amundsen et al. (1996) para cada embalse. (R: restos; P: pupas; L: larvas; O: otros). Feeding strategies according to the graphic method of Amundsen et al. (1996) for the four reservoirs sampled. (R: remains; $P$ : pupae; L: larvae; O: others).

accesibilidad. Por el contrario, el detrito representa un elemento de elevada constancia en la dieta de esta especie, y en general en todas las especies de barbos y todos los ecosistemas.

El detrito es un elemento muy común en la dieta de muchos peces de climas templados, especialmente en ciprínidos (Bowen, 1983), y en concreto en las especies de barbo, tanto en los sistemas lóticos como lénticos (Guillén, 1982; Granado-Lorencio \& García-Novo, 1986; Encina \& Granado-Lorencio, 1990, 1997; Magalhaes, 1992, 1993). Tambien en la mayoría de estos trabajos se refleja la importante incidencia en la dieta de este género del material vegetal (macrófitas, perifiton o elementos de origen terrestre, como cortezas, hojas o semillas). La posibilidad de explotación de este tipo de materiales permite maximizar la ingesta neta de energía, como demuestran los resultados de las estimas de ración diaria para el embalse de El Gergal $(\mathrm{RD}=$ $0,82 \%)$, con una elevada proporción de semillas, permitiendo un considerable descenso en el costo de la búsqueda del alimento en ambientes donde este recurso sea abundante. Al mismo tiempo puede tener un alto valor competitivo frente a otras especies bentófagas del ecosistema, o cuando los recursos tróficos son escasos o menores (como es el caso del invierno).

Existe una discrepancia en cuanto a las predicciones de la teoría del optimal foraging y la teoría general del nicho, respecto a la estrategia alimentícia desarrollada en situaciones de escasez de recursos tróficos. Mientras que en la primera predice que la alimentación es generalista cuando los recursos alimenticios son escasos (Pianka, 1981), la teoría del nicho predice que los individuos se especializan cuando las competencias intraespecíficas llegan a ser fuertes (es decir, bajo recursos limitantes). La teoría del optimal foraging a su vez predice una estrategia generalista con una alta componente intrafenotípica para la amplitud del nicho en dichas situaciones, mientras que la teoría del nicho predi- 
ce una estrategia alimenticia caracterizada por una especialización individualizada y una alta componente interfenotípica. Nuestros resultados concuerdan en todos los sentidos con las predicciones formuladas por la teoría del optimal foraging.

La versatilidad de los hábitos alimenticios del barbo, especialmente su estrategia trófica caracterizada por un elevado omnivorismo y generalismo, ha sido contemplado por la mayoría de los autores como una característica importante para la explotación de los sistemas lóticos ibéricos, en el cual las especies de este género han evolucionado, debido a la fluctuabilidad que caracteriza estos ecosistemas (Encina, 1991; Magalhaes, 1992, 1993; Encina \& Granado-Lorencio, 1997). Es esta misma versatilidad la que posiblemente haya contribuido decisivamente al éxito colonizador de estas especies en el nuevo ecosistema creado que supone el embalse, caracterizado igualmente, aunque en un sentido espacio-temporal bien distinto del sistema lótico, por marcadas fluctuaciones de los recursos tróficos. En relación a la alta componente intrafenotípica del nicho, también Magalhaes (1992) encontró para el barbo común (Barbus bocagei), que durante el invierno era la época en que la población mostraba la mayor uniformidad en la utilización de los recursos tróficos.

\section{AGRADECIMIENTOS}

El presente trabajo ha sido financiado por el Proyecto DIGICYT PB97-1198.

\section{BIBLIOGRAFÍA}

ALLEN, J. R. M. \& J. WOOTTON, 1982. Age, growth and rate of food consumption in an upland population of the three-spined stickleback, Gasterosteus aculeatus L. J. Fish. Biol., 21: 95-105.

AMEZAGA HERRÁN, R. 1988. Análisis de contenidos estomacales en peces. Revisión bibliográfica de los objetivos y la metodología. Informes Técnicos del Instituto Español de Oceanografía, $\mathrm{N}^{\circ}$ 63. Ministerio de Agricultura, Pesca y Alimentación. Madrid.
AMUNDSEN, P. A. 1995. Feeding strategy of Arctic charr (Salvelinus alpinus): general opportunist, but individual specialist. Nordic J. Freshw. Res. 71: 150-156.

AMUNDSEN, P. A., H. M. GABLER \& F. J. STALDVIK. 1996. A new approach to graphical analysis of feeding strategy from stomach contents data- modification of the Costello (1990) method. J. Fish Biol., 48: 607-614.

BOWEN, S. H. 1983. Detritivory in neotropical fish communities. Env. Biol. Fish., 9: 137-144.

ENCINA, L. 1986. Diferenciación merística y biométrica en el género Barbus: Estructura del aparato mandibular y segregación trófica. Tesis de Licenciatura. Univ. Sevilla. 241 pp.

ENCINA, L. 1991. Ecología trófica y dinámica energética de la comunidad íctica del río Guadalete. Tesis Doctoral. Univ. Sevilla. 327 pp.

ENCINA, L. \& C. GRANADO LORENCIO. 1988. Multivariate análisis of some morphometric characters in the genus Barbus (Pisces, Cyprinidae). Fol. Zool., 37: 273-288.

ENCINA, L. \& C. GRANADO LORENCIO. 1990. Morfoecología trófica en el género Barbus (Pisces, Cyprinidae). Limnetica, 6: 35-46.

ENCINA, L. \& C. GRANADO LORENCIO. 1991. Diet and diel feeding chronology of three iberian fish species. Ecology International Bulletin, 19: 43-64.

ENCINA, L. \& C. GRANADO LORENCIO. 1994. Gut evacuation in barbel (Barbus sclateri G. 1868) and nase (Chondrostoma willkommi S., 1866). Ecol. Freshwat. Fish., 23: 1-8.

ENCINA, L. \& C. GRANADO LORENCIO. 1997. Seasonal changes in condition, nutrition, gonad maturation and energy content in barbel, Barbus sclateri, inhabiting a fluctuating river. Environ. Biol. Fishes., 50: 75-84.

GUILLÉN, E. 1982. Estudio ecológico de la ictiofauna del Embalse de Torrejón (Río Tajo, Parque Natural del Monfragüe, Cáceres). Tesis de Licenciatura. Univ. Sevilla. 199 pp.

GRANADO-LORENCIO, C. 1983. Ecología de la comunidad íctica del embalse de Arrocampo (Cuenca del río Tajo, Cáceres). Tesis doctoral. Univ. Sevilla. 350 pp.

GRANADO-LORENCIO, C. \& F. GARCÍA-NOVO. 1986. Feeding habits of the fish community in a eutrophic reservoir in Spain. Ekol. Pol., 34: 95-110.

HYSLOP, E. J. 1980. Stomach content analysis: A review of methods and their application. J. Fish Biol., 17: 411-429. 
KAMIL, A. C., J. R. KREBS \& H. R. PULLIAM, 1987. Foraging Behavior. New York: Plenum Press.

KREBS, C. J. 1998. Ecological Methodology. $2^{\text {nd }}$ Ed. New York. Benjamin/Cummings. 620 pp.

LOBÓN-CERVIÁ, J. \& A. DIEGO. 1988. Feeding strategy of the barbel (Barbus bocagei Steind.) with relation to benthos composition. Arch. Hydrobiol., 114: 83-95.

MAGALHAES, M. F. 1992. Feeding ecology of the iberian cyprinid Barbus bocagei Steindachner, 1865 in a lowland river. J. Fish Biol., 40: 123-133.

MAGALHAES, M. F. 1993. Feeding of an Iberian stream cyprinid assemblage: seasonality of resources in a highly variable environment. Oecologia, 96: 253-260.

PENCZAK, T. 1990. Bioenergetic trends in ecological studies on fish in Poland. Pol. Ecol. Stud., 16 (3-4): 157-168.
PENCZAK, T. 1995. Food consumption by fish populations in the Wasta River, Poland, before and after impoundment. Hydrobiology, 302: 47-61.

PIANKA, E. R. 1981. Competition and niche theory. In: Theoretical Ecology. R.M. May (ed.): 167-196. Blackwell. Oxford.

SCHOENER, T. W. 1971. Theory of feeding strategies. Ann. Rev. Ecol. Syst., 2: 369-404.

STEWART, D. J., D. WEININGER, D. V. ROTTIERS \& T. A. EDSALL, 1983. An energetic model for lake trout, Salvelinus namaychus: Application to the lake Michigan population. Can. J. Fish. Aquat. Sci., 40: 681-698.

WINDELL, J. T. 1971. Food analyses and rate of digestion. In: Methods for assessment of fish production in freshwater. W.E. Ricker (ed.): 125-183. I.B.P. Handbook, No ${ }^{\circ}$. Blackwell. Oxford. 